VOLUME 22 (2017) 125-132

DOI: $10.24330 /$ ieja.325937

\title{
A NOTE ON THE FACTORIZATION OF PERMUTATIONS INTO CYCLES
}

\author{
J. Elder and O. Vega \\ Received: 27 December 2016; Revised: 14 April 2017 \\ Communicated by Burcu Üngör
}

\begin{abstract}
We find conditions on $k, n \in \mathbb{N}$, where $3 \leq k \leq n$ for which a permutation in $S_{n}$ can be written as a product of distinct $k$-cycles in $S_{n+i} \backslash S_{n}$, for some $i \in \mathbb{N}$. This result generalizes a problem that was solved in 2010 in an episode of the television show Futurama: the so-called Futurama Theorem.
\end{abstract}

Mathematics Subject Classification (2010): 20B30, 20F05

Keywords: Permutation, product of cycles, Futurama

\section{Introduction}

In the 2010 Writers Guild Award-winning episode of Futurama, The Prisoner of Benda [5], Professor Farnsworth and Amy build a machine that can swap the brains of any two people. The two use the machine to swap brains with each other, but then discover that once two people have swapped with each other, the machine does not swap them back. More characters get involved until the group is thoroughly mixed up, and they start looking for ways to return to their own heads.

We want to consider each brain swap done by the Professor's machine as a function that exchanges two elements in a set and fixes everything else: a transposition. Hence, we define $S_{n}$ to be the group of $n$ ! permutations of the set $\{1,2, \ldots, n\}$, and we adopt all standard notation about objects in $S_{n}$.

Now we can look at each brain swap as a transposition in $S_{n}$, where $n$ is the number of characters involved in the brain-swapping. Hence, the problem consists in writing the inverse of a permutation as a product of transpositions that (1) are all distinct, and (2) were not already used in constructing the original permutation.

The solution given in the Futurama episode relies on involving two additional people; these two characters were not involved in any of the original swaps, and so they have no restrictions on who they can swap brains with. For example, using

The first author thanks the Division of Graduate Studies and the College of Science and Mathematics at California State University, Fresno for her Robert \& Norma Craig Graduate Scholarship, and for travel support. 
the additional 'characters' $x$ and $y$, we have

$$
(12)^{-1}=\left(\begin{array}{lll}
x & y
\end{array}\right)(2 x)(1 y)(2 y)(1 x) .
$$

This can be done for any permutation, and leads to the following theorem.

Theorem 1.1 (Keeler, 2010). Let $n \in \mathbb{N}, n \geq 2$. The inverse of any permutation in $S_{n}$ can be written as a product of distinct transpositions in $S_{n+2} \backslash S_{n}$.

Although Ken Keeler (writer and executive producer for Futurama at the time) never published the proof of his theorem, the proof given in the episode as well as a write-up of the idea may be found in [3]. This was, most probably, the first time in which a theorem was proved for the purpose to advance the narrative of a TV show, and thus it made it to the (internet) news $[4,7]$ and was also mentioned in books, such as Simon Singh's The Simpsons and Their Mathematical Secrets [6]. Of course, this aroused the interest of mathematicians and, naturally, it did not take too long before articles were published about the problem (see [1] and [2]). Moreover, the solution given by Evans, Huang, and Nguyen in [1] is optimal in the sense that it uses the minimal number of cycles and the minimal number of additional elements. In that article, they also gave necessary and sufficient conditions on $m$ and $n$ for the identity permutation to be expressible as a product of $m$ distinct transpositions in $S_{n}$.

Although usually referred as The Futurama Theorem, in this work we will refer to Theorem 1.1 as Keeler's Theorem.

\section{Products of $k$-cycles, $k>2$}

Now we are interested in learning what would happen if Professor Farnsworth and Amy had created a machine that swapped several brains at once. We will assume that this machine contains a round table with $k$ chairs and that when $k$ people are seated around the table, the machine transfers each person's brain to the person on his/her right. For example, for $k=5$, the machine would do

$$
\text { Professor } \longrightarrow \text { Amy } \longrightarrow \text { Bender } \longrightarrow \text { Leela } \longrightarrow \text { Fry } \longrightarrow \text { Professor }
$$

The problem of undoing what the machine did in the example above would be easy if the machine did not have 'issues' and worked even when the same set of five characters sat around the machine for a second time; we could use that $\left(\begin{array}{lllll}1 & 2 & 3 & 4 & 5\end{array}\right)^{-1}=\left(\begin{array}{lllll}5 & 4 & 3 & 2 & 1\end{array}\right)$ or that $\left(\begin{array}{lllll}1 & 2 & 3 & 4 & 5\end{array}\right)^{-1}=\left(\begin{array}{lllll}1 & 2 & 3 & 4 & 5\end{array}\right)^{4}$ to obtain the inverse of the original permutation. That would be too easy, even for TV math. Hence, to make this interesting, if $\sigma$ is the $k$-cycle associated to a brain transfer 
that has been previously carried out, then the machine would not perform a brain transfer associated to $\sigma^{i}$, for all $i \in \mathbb{N}$. We will solve this problem in our main result (Theorem 2.2), after the following definitions and remark.

A permutation, $\sigma \in S_{n}$, is even if it can be written as the product of an even number of transpositions (including $\sigma=e$ ), otherwise permutation are odd. The set of all even permutations in $S_{n}$ is a subgroup of $S_{n}$ denoted $A_{n}$. Note that $k$-cycles are even/odd depending on $k$ being odd/even, respectively.

Remark 2.1. We are assuming that we are working with a machine that swaps cyclically the brains of $k$ people at a time. That is, we are working with $k$-cycles. Note that, if $k$ is odd then $k$-cycles are even permutations and thus our problem would reside in $A_{n}$.

Theorem 2.2. Let $n, k \in \mathbb{N}$ be such that $n>2, k \geq 3$, and $n \geq k$. If $\sigma \in S_{n}$, then, $\sigma^{-1}$ can be written as a product of $k$-cycles

$$
\sigma^{-1}=\tau_{1} \tau_{2} \cdots \tau_{t}
$$

under the following conditions

- if $k=3$ and $\sigma \in A_{n}$, then $\tau_{i} \in A_{n+1} \backslash A_{n}$.

- if $k>3$ and $\sigma \in A_{n}$, then $\tau_{i} \in S_{n+(k-3)} \backslash S_{n}$.

- if $k=2 j$, for some $j \in \mathbb{N}$, and $\sigma \in S_{n} \backslash A_{n}$, then $\tau_{i} \in S_{n+3(j-1)} \backslash S_{n}$.

for all $i=1,2, \ldots, t$, and $\tau_{i} \notin<\tau_{j}>$, for all $i \neq j$.

The proof of Theorem 2.2 follows from Theorem 2.5, Theorem 2.6, and Theorem 2.7. We will start our study with what will end up being an interesting special case: $k=3$.

2.1. Products of 3-cycles. It turns out that, in this case, we only need one extra element to construct the inverse of any permutation in $A_{n}$.

Lemma 2.3. Let $n \in \mathbb{N}, n>2$, and let $\sigma \in A_{n}$ be a $k$-cycle, for some odd $k>1$. Then, $\sigma^{-1}$ can be written as a product of 3 -cycles

$$
\sigma^{-1}=\tau_{1} \tau_{2} \cdots \tau_{t}
$$

where $\tau_{i} \in A_{n+1} \backslash A_{n}$, for all $i=1,2, \ldots, t$, and $\tau_{i} \notin<\tau_{j}>$, for all $i \neq j$.

Proof. For $k=3$ we get $\left(\begin{array}{lll}1 & 2 & 3\end{array}\right)=\left(\begin{array}{lll}x & 3 & 1\end{array}\right)\left(\begin{array}{lll}x & 1 & 2\end{array}\right)$, and for every $3<k \leq n, k$ odd, and $x>n$, consider the product

$$
(12 \cdots k-1 k)=(x k 1)(x k-2 k-1) \cdots(x 34)(x 12) .
$$


The result is now immediate, as the inverse of any odd cycle is also an odd cycle, and the 3-cycles used in the product fix different sets of elements.

Lemma 2.4. Let $\alpha, \beta \in S_{n}, n>2$, be two disjoint even cycles. Then, $(\alpha \beta)^{-1}$ can be written as a product of 3-cycles

$$
(\alpha \beta)^{-1}=\tau_{1} \tau_{2} \cdots \tau_{t}
$$

where $\tau_{i} \in A_{n+1} \backslash A_{n}$, for all $i=1,2, \ldots, t$, and $\tau_{i} \notin<\tau_{j}>$, for all $i \neq j$.

Proof. Let $\beta^{-1}=\left(\begin{array}{llll}a_{1} & a_{2} & \cdots & a_{r}\end{array}\right)$ and $\alpha^{-1}=\left(\begin{array}{llll}b_{1} & b_{2} & \cdots & b_{s}\end{array}\right)$ be two disjoint cycles, where $r$ and $s$ are even. Note that $(\alpha \beta)^{-1}=\beta^{-1} \alpha^{-1} \in A_{n}$ can be written as

$$
\begin{aligned}
\beta^{-1} \alpha^{-1} & =\left(\begin{array}{lll}
a_{1} & a_{2}
\end{array}\right)\left(\begin{array}{llll}
b_{1} & b_{2}
\end{array}\right)\left(a_{2} a_{3} \cdots a_{r}\right)\left(b_{2} b_{3} \cdots b_{s}\right) \\
& =\left(\begin{array}{llll}
b_{2} & b_{1} & x
\end{array}\right)\left(b_{1} a_{2} x\right)\left(a_{2} a_{1} x\right)\left(a_{1} b_{1} x\right)\left(a_{2} a_{3} \cdots a_{r}\right)\left(b_{2} b_{3} \cdots b_{s}\right) .
\end{aligned}
$$

Note that the last two cycles in the product are odd, and thus we can write them as a product of 3-cycles, as indicated in Lemma 2.3. It is easy to see now that $(\alpha \beta)^{-1}$ can be written as claimed.

We summarize the previous two lemmas, and make explicit our main result for $k=3$, in the following theorem.

Theorem 2.5. Let $\sigma \in A_{n}, n>2$. Then, $\sigma^{-1}$ can be written as a product of 3-cycles

$$
\sigma^{-1}=\tau_{1} \tau_{2} \cdots \tau_{t}
$$

where $\tau_{i} \in A_{n+1} \backslash A_{n}$, for all $i=1,2, \ldots, t$, and $\tau_{i} \notin<\tau_{j}>$, for all $i \neq j$.

Proof. Given $\sigma \in A_{n}$, we know we can write $\sigma^{-1}$ as a product of disjoint cycles. Moreover, after rearrangement (if needed) we can put all the appearing even cycles (if any) in pairs. Hence, Lemmas 2.3 and 2.4 guarantee the desired factorization of $\sigma^{-1}$.

2.2. The case $\sigma \in A_{n}$ with $k>3$. Now we want to learn what would happen if the Professor's machine swapped $k$ brains cyclically, where $k>3$, and the end result of all the brain-swapping ended up being represented by an even permutation. In order to do that we need to set some notation.

Fix $n, k \in \mathbb{N}$, where $k>3$ and $n>2$. We define the following ordered lists of numbers

$$
\begin{aligned}
& {[x]_{n, k}=x_{1} x_{2} \ldots x_{k-3}} \\
& {[x]_{n, k}^{-1}=x_{k-3} \ldots x_{2} x_{1}}
\end{aligned}
$$


where $x_{i} \in \mathbb{N}$ and $x_{i}>n$, for all $i=1,2, \ldots, k-3$, and $x_{i} \neq x_{j}$, for all $i \neq j \in$ $\{1,2, \ldots, k-3\}$. We will use these lists to write cycles. For example $\left(12[x]_{3,7}\right)=$

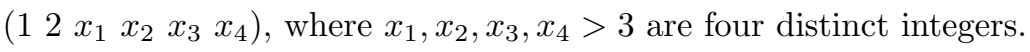

Theorem 2.6. Let $n, k \in \mathbb{N}$ be such that $n>2$ and $k>3$. If $\sigma \in A_{n}$, then, $\sigma^{-1}$ can be written as a product of $k$-cycles

$$
\sigma^{-1}=\tau_{1} \tau_{2} \cdots \tau_{t}
$$

where $\tau_{i} \in S_{n+(k-3)} \backslash S_{n}$, for all $i=1,2, \ldots, t$, and $\tau_{i} \notin<\tau_{j}>$, for all $i \neq j$.

Proof. We will first prove that every element $\sigma \in A_{n}$ can be written as a product of $k$-cycles, then we will prove that the factors used satisfy the conditions claimed in the theorem.

We know that every element in $A_{n}$ can be written as a disjoint product of odd cycles and an even number of even cycles. We will first deal with $u$-cycles for odd $u>1$. For $u=3$ we can write

$$
\left(\begin{array}{lll}
1 & 2 & 3
\end{array}\right)=\left(\begin{array}{llll}
1 & 3 & 2 & {[x]_{n, k}^{-1}}
\end{array}\right)\left(\begin{array}{llll}
2 & 1 & 3 & {[x]_{n, k}}
\end{array}\right),
$$

and so we need $k-3$ new elements to write a 3 -cycle as a product of $k$-cycles. For $u>3$ we know that every $u$-cycle in $A_{n}$ can be written as a product of $\frac{u-1}{2}$ distinct 3-cycles as follows:

$$
\left(\begin{array}{llll}
a_{1} & a_{2} & \cdots & a_{u}
\end{array}\right)=\left(\begin{array}{llll}
a_{1} & a_{2} & a_{3}
\end{array}\right)\left(a_{3} a_{4} a_{5}\right) \cdots\left(a_{u-2} a_{u-1} a_{u}\right) .
$$

So, if $\sigma \in A_{n}$ is a $u$-cycle with $u>3$, then we write it as in Equation (2). After that we write each of the 3 -cycles in that product as a product of two $k$-cycles, as discussed in Equation (1) above. Hence, we can write $\sigma$ as a product of $k$-cycles by incorporating $k-3$ new elements.

For products of two even cycles we let $\alpha=\left(\begin{array}{llll}b_{1} & b_{2} & \cdots & b_{r}\end{array}\right)$ and $\beta=\left(\begin{array}{llll}c_{1} & c_{2} & \cdots & c_{s}\end{array}\right)$, where $r$ and $s$ are even. Note that $\alpha \beta \in A_{n}$ can be written as

$$
\begin{aligned}
& \alpha \beta=\left(b_{1} b_{2}\right)\left(c_{1} c_{2}\right)\left(b_{2} b_{3} \cdots b_{r}\right)\left(c_{2} c_{3} \cdots c_{s}\right) \\
& =\left(\begin{array}{llll}
c_{2} & c_{1} & b_{2} & {[x]_{n, k}^{-1}}
\end{array}\right)\left(b_{2} b_{1} c_{1}\left[\begin{array}{ll}
x]_{n, k}
\end{array}\right)\left(b_{2} b_{3} \cdots b_{r}\right)\left(c_{2} c_{3} \cdots c_{s}\right)\right.
\end{aligned}
$$

where the first two cycles needed $k-3$ new elements to have length $k$, and all the other cycles involved are odd, and thus can be written as products of $k$-cycles.

In order to see that all the $k$-cycles obtained in the product describing $\sigma^{-1}$ generate distinct subgroups of $A_{n+(k-3)}$ we start with the two $k$-cycles in Equation (1). These cycles, say $\tau_{1}=\left(\begin{array}{llll}1 & 3 & 2 & {[x]_{n, k}^{-1}}\end{array}\right)$ and $\tau_{2}=\left(\begin{array}{llll}2 & 1 & 3 & {[x]_{n, k}}\end{array}\right)$, generate distinct subgroups of $A_{n}$ because, although they 'move' the same set of $k$ elements, if they 
did not then we would get $\tau_{2}^{i}=\tau_{1}$, for some $i$. However, if we look at $\tau_{2}^{i}(2)$ we can see that the only possibility for $\tau_{2}^{i}=\tau_{1}$ to happen would be when $i=k-1$, but it is easy to see that $\tau_{2}^{k-1}=\tau_{2}^{-1} \neq \tau_{1}$.

Next we notice that the $k$-cycles obtained by applying Equation (1) to each of the 3 -cycles in Equation (2) either come in pairs as in Equation (1) (already discussed), or are $k$-cycles that 'move' different sets of elements; in the latter case we get that the groups generated by such cycles intersect trivially, as no power, $i=1, \ldots, k-1$, of a $k$-cycle $\tau$ can fix any of the $k$ elements that $\tau$ does not fix.

Now, if we took Equation (3) and applied the previous two paragraphs to it we would get that $\left(b_{2} b_{3} \cdots b_{r}\right)\left(c_{2} c_{3} \cdots c_{s}\right)$ is written into $k$-cycles that generate distinct subgroups. These subgroups are also distinct to the ones generated by $\left(\begin{array}{llll}c_{2} & c_{1} & b_{2} & {[x]_{n, k}^{-1}}\end{array}\right)$ and $\left(\begin{array}{lllll}b_{2} & b_{1} & c_{1} & {[x]_{n, k}}\end{array}\right)$, as the latter $k$-cycles 'move' a different set of elements than the $k$--cycles in the factorization of $\left(\begin{array}{llll}b_{2} & b_{3} & \cdots & b_{r}\end{array}\right)\left(\begin{array}{llll}c_{2} & c_{3} & \cdots & c_{s}\end{array}\right)$. We get that all the $k$-cycles obtained in the factorization of $\alpha \beta$ generated distinct subgroups.

It is immediate that a permutation $\tau$ yields a product of $k$-cycles (when written as a product of $k$-cycles as described above) that do not 'move' the same $k$-elements as any of the $k$-cycles in the product of a permutation that is disjoint to $\tau$. This finishes the proof, as with this we are able to write the inverse of any permutation as a disjoint product of cycles, and these cycles can be dealt with separate from the rest without running the risk of getting any $k$-cycles appearing more than once in the final product.

2.3. The case $\sigma \in S_{n} \backslash A_{n}$, with even $k>2$. Next we study the last case needed to complete the proof of Theorem 2.2 .

Theorem 2.7. Let $n, j \in \mathbb{N}$ be such that $n>2$ and $j>1$. If $\sigma \in S_{n} \backslash A_{n}$, then, $\sigma^{-1}$ can be written as a product of $(2 j)$-cycles

$$
\sigma^{-1}=\tau_{1} \tau_{2} \cdots \tau_{t}
$$

where $\tau_{i} \in S_{n+3(j-1)} \backslash S_{n}$, for all $i=1,2, \ldots, t$, and $\tau_{i} \notin<\tau_{k}>$, for all $i \neq k$.

Proof. Let $\sigma \in S_{n} \backslash A_{n}$. We write $\sigma^{-1}$ as a product of disjoint cycles and we let $\sigma_{1}=\left(\begin{array}{llll}a_{1} & a_{2} & \cdots & a_{r}\end{array}\right)$ be an even cycle in this decomposition. We next factor $\sigma$ as follows

$$
\sigma_{1}=\left(\begin{array}{ll}
a_{1} & a_{2}
\end{array}\right)\left(a_{2} \cdots a_{r}\right) .
$$

Hence, we can always write $\sigma=\left(a_{1} a_{2}\right) \tau$, where $\tau \in A_{n}$ and $\tau\left(a_{1}\right)=a_{1}$. 
Theorem 2.6 helps us to write $\tau$ as a product of $(2 j)$-cycles satisfying the conditions in this lemma. For the transposition we proceed as how we did for Theorem 2.6 , by first defining the following lists

$$
\begin{aligned}
& {[x]=x_{1} x_{2} \ldots x_{j-1} \quad[x]^{-1}=x_{j-1} \ldots x_{2} x_{1}} \\
& {[y]=y_{1} y_{2} \ldots y_{j-1} \quad[y]^{-1}=y_{j-1} \ldots y_{2} y_{1}} \\
& {[z]=z_{1} z_{2} \ldots z_{j-1} \quad[z]^{-1}=z_{j-1} \ldots z_{2} z_{1}}
\end{aligned}
$$

where $x_{i}, y_{i}, z_{i} \gg n$, for all $1 \leq i \leq j-1$. We now notice that

$$
\left(a_{1} a_{2}\right)=\left(a_{1}[z]^{-1}[y]^{-1} a_{2}\right)\left(a_{1}[x]^{-1} a_{2}[z]\right)\left([x] a_{1}[y] a_{2}\right)
$$

is a product of $(2 j)$-cycles.

Finally, we need to check that the groups generated by the $(2 j)$-cycles intersect trivially. We already have that for the cycles in the factorization of $\tau$, and the same can be easily obtained for the cycles in Equation (4), as the three cycles in this product 'move' different sets of elements. A similar argument can be used when checking subgroups generated by the $(2 j)$-cycles in the product describing $\tau$ and those in the product in Equation (4).

Acknowledgment. The authors would like to sincerely thank the referee for all the valuable suggestions and comments; they really helped to get a better exposition of our work. We believe the article benefited substantially from their comments.

\section{References}

[1] R. Evans, L. Huang and T. Nguyen, Keeler's theorem and products of distinct transpositions, Amer. Math. Monthly, 121(2) (2014), 136-144.

[2] R. Evans and L. Huang, Mind switches in Futurama and Stargate, Math. Mag., 87(4) (2014), 252-262.

[3] H. Georgiev, The Futurama theorem explained, The Commutator, 2 (2010), $18-20$.

[4] T. Phillips, Math in the Media, Amer. Math. Soc., Original math on Futurama, (2010).

http://www . ams.org/news/math-in-the-media/10-2010-media.

[5] Previous Nominees \& Winners of the Writers Guild Awards (last accessed on 08/15/16).

http: //awards . wga.org/wga-awards/previous-nominees-winners.

[6] S. Singh, The Simpsons and their mathematical secrets, Bloomsbury, NY, 2013. 
[7] The prisoner of Benda, The Infosphere, the Futurama Wiki (last accessed on 08/15/16).

http://theinfosphere.org/The_Prisoner_of_Benda.

\section{J. Elder}

Department of Mathematics

Arizona State University, Tempe

AZ 85287, USA

e-mail: Jennifer.E.Elder@asu.edu

O. Vega (Corresponding Author)

Department of Mathematics

California State University, Fresno

Fresno, CA 93740, USA

e-mail: ovega@csufresno.edu 